\title{
Storage and Dissipation Limits in Resonant Switched-Capacitor Converters
}

\author{
Jonathan C. Mayo-Maldonado ${ }^{D},{ }^{1}$ T. M. Maupong, ${ }^{2}$ \\ Jesús E. Valdez-Reséndiz, ${ }^{1}$ and Julio C. Rosas-Caro ${ }^{3}$ \\ ${ }^{1}$ School of Engineering and Sciences, Tecnologico de Monterrey, Monterrey, NL, Mexico \\ ${ }^{2}$ College of Sciences, Botswana International University of Science and Technology, Palapye, Botswana \\ ${ }^{3}$ Engineering Faculty, Universidad Panamericana, Guadalajara, JAL, Mexico
}

Correspondence should be addressed to Jonathan C. Mayo-Maldonado; jcmayo@itesm.mx

Received 7 July 2017; Revised 10 November 2017; Accepted 26 November 2017; Published 24 January 2018

Academic Editor: J.-C. Cortés

Copyright (C) 2018 Jonathan C. Mayo-Maldonado et al. This is an open access article distributed under the Creative Commons Attribution License, which permits unrestricted use, distribution, and reproduction in any medium, provided the original work is properly cited.

\begin{abstract}
The purpose of this manuscript is twofold, first we introduce an energy-based modeling framework for the analysis of resonant switched-capacitor (SC) converters and second we demonstrate that energy storage and dissipation in resonant SC with ideal switches are bounded by a fundamental physical limit that, up until now, has been only associated with the special case of pure SC topologies. For instance, we show that the maximum energy stored in the small size inductors in resonant SC converters is equal to the energy that would be dissipated by their purely SC counterpart. The presented analysis permits the computation of resonant inductances in terms of maximum current peak values, which is experimentally validated. Furthermore, we introduce a relative loss factor that permits determining the efficiency of a design for a general case in the presence of parasitic resistances. These results corroborate that migrating to resonant SC technologies is one of the most compelling alternatives to overcome well-known disadvantages in pure SC topologies.
\end{abstract}

\section{Introduction}

DC-DC power converters with switched-capacitors exhibit highly desirable features in energy conversion systems such as high-voltage gains, high-efficiency, and transformerless profiles (see, e.g., [1-5]). An important issue in SC converters is its dynamic analysis, since while state-space modeling techniques such as state averaging are effective for some traditional classes of power converters, SC converters cannot be modeled in this way. Although the modeling of standard DC-DC converters is conventionally carried out considering ideal switches, parallel connections between capacitors in SC converters induce voltage discontinuities (see $[1,6]$ ), demanding a more refined analysis (see [7]).

Another important challenge in SC converters is their efficiency, for which many approaches and studies have been proposed; see, for instance, [8-11], where losses due to the charging/discharging process of capacitors have been identified besides the standard conduction losses. Moreover, these topologies exhibit also high current peaks due to sudden parallel interconnection of capacitances, demanding high stress on semiconductor devices. For this reason, the use of small inductances that limit such currents is a common alternative (see [12-16]). The justification for the use of such limiting current inductors is mainly based on their size and cost, since resonant inductors are not required to store a significant amount of energy as in other traditional topologies.

In this paper, we introduce a modeling framework for resonant SC and pure SC converters based on switched linear differential systems (see [17-19]). Moreover, a natural, modular way to describe power and energy as quadratic quantities is introduced using the calculus of quadratic differential forms [20]. This setting is the pivotal figure in our ensuing results that encompass the study of efficiency and performance issues in resonant SC topologies, with respect to pure SC converters. Previous contributions that elaborate on these issues include [13], where a study of 
conduction losses computation is presented. In [15], the authors argue that charging/discharging losses are mitigated in resonant SC topologies and consequently conduction losses are predominant. This contribution is well-supported by interesting discussions and results. In [21], the authors present an extension of the SC converter energy losses analysis, based on the so-called slow- and fast-switching limits, to the case of resonant SC converters. Motivated by the current trends in the study of losses in SC resonant converters, we show a rigorous analytical proof that corroborates that any loss in resonant SC converters must be regarded as conduction losses, due to parasitic (or ESR) resistors. We also generalize the results in resonant SC and pure SC converters involving parasitic resistances by proving that losses are upper bounded by a fundamental physical limit.

The results presented in this paper are not straightforwardly evident nor reached by pure intuition since, for instance, it is well-known that SC converters with ideal switches dissipate energy at switching instants (even when parasitic resistances are neglected), which means that resonant SC converters with negligible inductances are also lossy. Consequently the effect of adding a tiny inductor originally considered to damp peak currents that is by observation, as reported in [15], mitigating energy transfer (discharging) losses, deserves a clear explanation. The study of such energy conservation mechanism is adopted as the main conviction in this paper, while the advantages of the results are illustrated with the design of resonant SC cells with respect to peak value specifications, which is experimentally validated and a relative loss factor that permits computing losses in a general case.

In this paper, we use the following notation. The space of $\mathrm{w}$-dimensional real vectors is denoted by $\mathbb{R}^{\mathrm{w}}$ and that of $\mathrm{g} \times \mathrm{w}$ real matrices is denoted by $\mathbb{R}^{\mathrm{g} \times \mathrm{w}}$; when one of the dimensions is not specified we use $\bullet ; \operatorname{col}(A, B)$ denotes the matrix obtained by stacking the matrix $A \in \mathbb{R}^{\bullet \times w}$ over $B \in \mathbb{R}^{\bullet \times w}$. The ring of polynomials with real coefficients in the indeterminate $s$ is denoted by $\mathbb{R}[s]$; the ring of two-variable polynomials with real coefficients in the indeterminates $\zeta$ and $\eta$ is denoted by $\mathbb{R}[\zeta, \eta] . \mathbb{R}^{\mathrm{r \times w}}[s]$ denotes the set of all $r \times w$ matrices with entries in $s$ and $\mathbb{R}^{\mathrm{n} \times \mathrm{m}}[\zeta, \eta]$ that of $\mathrm{n} \times \mathrm{m}$ polynomial matrices in $\zeta$ and $\eta \cdot I_{\mathrm{w}}$ denotes an identity matrix of dimensions $\mathrm{w} \times \mathrm{w} ; A^{\top}$ denotes the transpose the matrix $A$. Let $\tau \in \mathbb{R}$ and $f: \mathbb{R} \rightarrow \mathbb{R}^{\mathrm{w}}$; then we define $f\left(t^{+}\right):=$ $\lim _{\tau \searrow t} f(\tau)$ and $f\left(t^{-}\right):=\lim _{\tau>t} f(\tau)$, provided that such limits exist.

\section{Higher-Order Modeling}

When modeling from first principles, we usually focus on a set of variables of interest (e.g., input/output voltages and currents) that are important for analysis, control, simulation, and so on. Occasionally, we need to introduce some auxiliary variables (e.g., nodal voltages, mesh currents, and state variables) that permit writing first principle equations in a convenient way. In this paper, we denote a vector of variables of interest by $w:=\operatorname{col}\left(w_{1}, w_{2}, \ldots, w_{\mathrm{w}}\right)$ and that of auxiliary variables by $z:=\operatorname{col}\left(z_{1}, z_{2}, \ldots, z_{\mathrm{z}}\right)$.
In general, during the modelling stage we obtain sets of linear differential equations (e.g., by applying first principles, by algebraic elimination of variables, and by using the calculus of impedances) of the form

$$
G_{0} w+\cdots+G_{L} \frac{d^{L}}{d t^{L}} w=M_{0} z+\cdots+M_{N} \frac{d^{N}}{d t^{N}} z,
$$

with $G_{i} \in \mathbb{R}^{\mathrm{p} \times \mathrm{w}}, i=0, \ldots, L$, and $M_{j} \in \mathbb{R}^{\mathrm{p} \times \mathrm{z}}, j=0, \ldots, N$. This set of equations can be written in a compact form as

$$
G\left(\frac{d}{d t}\right) w=M\left(\frac{d}{d t}\right) z
$$

where $G \in \mathbb{R}^{\mathrm{p} \times(L+1) \mathrm{w}}[s]$ and $M \in \mathbb{R}^{\mathrm{p} \times(N+1) \mathrm{z}}[s]$, that is, $G$ and $M$ are polynomial matrices, and thus are defined as

$$
\begin{aligned}
G(s) & :=G_{0}+G_{1} s+\cdots+G_{L} s^{L}, \\
M(s) & :=M_{0}+M_{1} s+\cdots+M_{N} s^{N} .
\end{aligned}
$$

A special case of (2) arises when $G$ equals the identity; that is,

$$
w=M\left(\frac{d}{d t}\right) z
$$

Equation (4) is called image representation and it can be always achieved when the system under analysis is controllable (see [22], Section 6.6, pp. 234-236).

An image representation can be also conveniently associated with the concept of transfer function, that is, a representation of the form $Y(s) U(s)^{-1}$, where $Y$ and $U$ are polynomial matrices of suitable rank and dimensions. Consider an inputoutput partition of the external variables of (4), that is, $w=$ $\operatorname{col}(u, y)$, possibly permuting the components of $w$. Consequently the matrix $M$ is partitioned accordingly as (here we assume that $Y(s) U(s)^{-1}$ has no pole/zero cancellations; see [22], Section 3.3 and Theorem 5.3.3)

$$
\underbrace{\left[\begin{array}{l}
u \\
y
\end{array}\right]}_{=w}=\underbrace{\left[\begin{array}{c}
U\left(\frac{d}{d t}\right) \\
Y\left(\frac{d}{d t}\right)
\end{array}\right]}_{=M(d / d t)} z,
$$

where the number of components in $z$ is the same as those in $u$ (see [23], Section VI-A). Moreover, in the case of imittances, the inputs $u=U(d / d t) z$ and outputs $y=Y(d / d t) z$ are conjugate variables, for example, pairs of input/output port voltages and currents; consequently they have the same number of components.

Example 1. Consider the circuit in Figure 1 where the variables of interest are $v$ and $i_{1}$ that correspond to the input voltage and the current through inductor $L_{1}$, respectively.

This circuit can be modelled as an impedance $Z(s)$, by standard series/parallel reductions; that is,

$$
\begin{aligned}
Z(s) & =\frac{v(s)}{i_{1}(s)}=L_{1} s+\frac{\left(L_{2} s+R\right)(1 / C s)}{\left(L_{2} s+R\right)+(1 / C s)} \\
& =\frac{L_{1} L_{2} C s^{3}+R L_{1} C s^{2}+\left(L_{1}+L_{2}\right) s+R}{L_{2} C s^{2}+R C s+1} .
\end{aligned}
$$




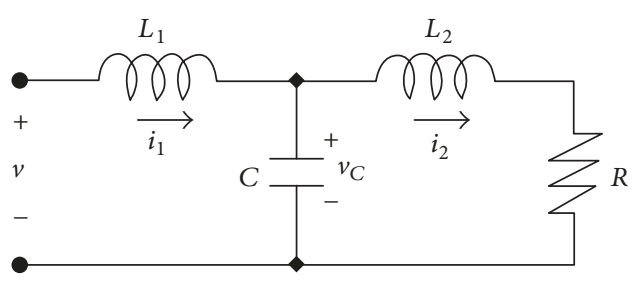

FIGURE 1: Electrical circuit.

The circuit admits an image representation

$$
\begin{aligned}
& {\left[\begin{array}{l}
v \\
i_{1}
\end{array}\right]} \\
& =\underbrace{\left[\begin{array}{c}
L_{1} L_{2} C \frac{d^{3}}{d t^{3}}+R L_{1} C \frac{d^{2}}{d t^{2}}+\left(L_{1}+L_{2}\right) \frac{d}{d t}+R \\
L_{2} C \frac{d^{2}}{d t^{2}}+R C \frac{d}{d t}+1
\end{array}\right]}_{M(d / d t)} i_{2},
\end{aligned}
$$

where, as previously mentioned, $w:=\operatorname{col}\left(v, i_{1}\right)$ is the vector of variables of interest and $z:=i_{2}$ is an auxiliary variable.

\section{Higher-Order Functionals}

In the study of electrical circuits we are very often not only interested in the analysis of the system variables, such as voltages/currents, but also interested in the study of some functionals of those variables, such as power and energy. Quadratic functionals for higher-order differential systems can be expressed in terms of quadratic differential forms (QDFs) induced by two-variable polynomial matrices (see [20]). Such polynomial characterization brings relevant algorithmic advantages that will be continuously exploited in this paper. In the following, and as a standing assumption in the rest of the paper, we assume that QDFs act only on infinitely differentiable trajectories, whose set is denoted by $\mathfrak{c}^{\infty}\left(\mathbb{R}, \mathbb{R}^{\bullet}\right)$, avoiding the presence of dirac impulses and their derivatives.

3.1. Algebraic Parametrization. Let $\Phi \in \mathbb{R}^{\mathrm{w} \times \mathrm{w}}[\zeta, \eta]$; then

$$
\Phi(\zeta, \eta):=\sum_{h, k} \Phi_{h, k} \zeta^{h} \eta^{k}
$$

with $\Phi_{h, k} \in \mathbb{R}^{\mathrm{w} \times \mathrm{W}}$ inducing the quadratic differential form (QDF) defined by

$$
Q_{\Phi}(w):=\sum_{h, k}\left(\frac{d^{h} w}{d t^{h}}\right)^{\top} \Phi_{h, k} \frac{d^{k} w}{d t^{k}} .
$$

Note that the variables $\zeta$ and $\eta$ act as placeholders for the derivatives of $w$ and their transpose.

Associated with $\Phi \in \mathbb{R}^{\mathrm{w} \times \mathrm{w}}[\zeta, \eta]$ is a coefficient matrix defined by $\widetilde{\Phi}:=\left[\Phi_{h, k}\right]_{h, k=0,1,2, \ldots}$. Note that since $Q_{\Phi}$ acts on infinitely differential trajectories, the matrix $\widetilde{\Phi}$ is an infinite matrix with only a finite number of nonzero entries (cf. [20], p. 1708). Moreover, $\Phi(\zeta, \eta)$ is called symmetric if $\Phi(\zeta, \eta)=$ $\Phi(\eta, \zeta)^{\top}$, or equivalently if $\widetilde{\Phi}=\widetilde{\Phi}^{\top}$; in the rest of the paper we will focus on the symmetric case.

A relevant advantage in the calculus of QDFs is their differentiation, which is a straightforward matter in terms of two-variable polynomial matrices. For instance, the derivative of $Q_{\Psi}$ is the $\mathrm{QDF} Q_{\Phi}$ defined by

$$
Q_{\Phi}(w):=\frac{d}{d t}\left(Q_{\Psi}(w)\right) \quad \forall w \in \mathfrak{C}^{\infty}\left(\mathbb{R}, \mathbb{R}^{w}\right) .
$$

In terms of two-variable polynomials, this derivative holds if and only if (see [20], p. 1710)

$$
\Phi(\zeta, \eta)=(\zeta+\eta) \Psi(\zeta, \eta)
$$

In brief, this approach shows that while sets of linear differential equations are most conveniently associated with one-variable polynomial matrices, quadratic functionals are best characterized in terms of two-variable ones.

3.2. Positivity, Negativity, and Reformulation in Terms of Auxiliary Variables. $Q_{\Phi}$ is nonnegative with respect to (4) if $Q_{\Phi}(w) \geq 0$ for all $w$ that satisfies $(4)$ with $w \in \mathbb{C}^{\infty}\left(\mathbb{R}, \mathbb{R}^{\mathrm{W}}\right)$ and positive with respect to $(4)$ if $Q_{\Phi}(w)>0$ for all $w$ that satisfies (4) with $w \in \mathfrak{C}^{\infty}\left(\mathbb{R}, \mathbb{R}^{\mathrm{w}}\right)$ and $\left[Q_{\Phi}(w)=0\right] \Rightarrow[w=0]$. The concepts of nonpositive and negative QDF are defined analogously.

QDFs can be reformulated in terms of the auxiliary variable, which simplifies certain computations including positivity and negativity tests. Given $\Phi \in \mathbb{R}^{\mathrm{w} \times \mathrm{w}}[\zeta, \eta]$, if $w$ and $z$ are related to $(4)$, defining

$$
\Phi^{\prime}(\zeta, \eta):=M(\zeta)^{\top} \Phi(\zeta, \eta) M(\eta)
$$

implies $Q_{\Phi}^{\prime}(z)=Q_{\Phi}(w)$, and consequently it follows that $Q_{\Phi} \geq 0$ for $w$ satisfying (4) if and only if $Q_{\Phi^{\prime}} \geq 0$ on $\mathfrak{C}^{\infty}\left(\mathbb{R}, \mathbb{R}^{z}\right)$. We adopt the notation $Q_{\Phi}$ to refer to a QDF acting on the external variable and $Q_{\Phi^{\prime}}$, to denote the associated QDFs acting on auxiliary variables.

\section{Energy-Based Analysis}

We now study energy functions in electrical systems. We afterwards extend this analysis to SC converters.

As previously illustrated, external variables in port circuits can be denoted by $w=\operatorname{col}(V, I)$, where $V$ and $I$ are vectors of conjugate variables (input voltages and currents, resp.) with the same number $m$ of components. For instance, the input power describing the energy flow into a circuit is

$$
Q_{\Phi}(w):=\frac{1}{2} w^{\top}\left[\begin{array}{cc}
0 & I_{m} \\
I_{m} & 0
\end{array}\right] w=V^{\top} I .
$$

By using (4), we can equivalently compute

$$
\begin{aligned}
Q_{\Phi}(w) & =Q_{\Phi^{\prime}}(z) \\
& :=\frac{1}{2}\left(M\left(\frac{d}{d t}\right) z\right)^{\top}\left[\begin{array}{cc}
0 & I_{m} \\
I_{m} & 0
\end{array}\right] M\left(\frac{d}{d t}\right) z .
\end{aligned}
$$


A fundamental principle in passive circuits states that the rate of change of energy stored inside the circuit, denoted by $Q_{\Psi}$, is never greater than the power that is being supplied; that is,

$$
Q_{\Phi} \geq \frac{d}{d t} Q_{\Psi} ; \quad \text { with } Q_{\Psi} \geq 0
$$

This means that a portion of the supplied energy has been stored in the circuit, while the rest of it has been dissipated. If we denote such dissipation by $Q_{\Delta}$, the above inequality yields

$$
Q_{\Phi}=\frac{d}{d t} Q_{\Psi}+Q_{\Delta},
$$

where $Q_{\Delta} \geq 0$ is the energy dissipated as heat by resistors.

Example 2. Consider the circuit in Figure 1 where $w$ := $\operatorname{col}\left(v, i_{1}\right)$ and $z:=i_{2}$ is an auxiliary variable. Using (7), we define the input power as in (14). After some straightforward computations we obtain

$$
\begin{aligned}
v i_{1}= & R i_{2}^{2}+\frac{1}{2} L_{1} \frac{d}{d t}\left(L_{2} C \frac{d^{2}}{d t^{2}} i_{2}+R C \frac{d}{d t} i_{2}+i_{2}\right)^{2} \\
& +\frac{1}{2} C \frac{d}{d t}\left(L_{2} \frac{d}{d t} i_{2}+R i_{2}\right)^{2}+\frac{1}{2} L_{2} \frac{d}{d t} i_{2}^{2} .
\end{aligned}
$$

Since

$$
\begin{aligned}
& i_{1}=L_{2} C \frac{d^{2}}{d t^{2}} i_{2}+R C \frac{d}{d t} i_{2}+i_{2} ; \\
& v=L_{2} \frac{d}{d t} i_{2}+R i_{2} ;
\end{aligned}
$$

then (17) is equivalent to

$$
\begin{aligned}
\underbrace{v i_{1}}_{\text {Input Power }\left(\mathrm{Q}_{\Phi}\right)}= & \frac{d}{d t} \underbrace{\left(\frac{1}{2} L_{1} i_{1}^{2}+\frac{1}{2} C v^{2}+\frac{1}{2} L_{2} i_{2}^{2}\right)}_{\text {Stored Energy }\left(\mathrm{Q}_{\Psi}\right)} \\
& +\underbrace{R i_{2}^{2}}_{\text {Dissipation }\left(\mathrm{Q}_{\Delta}\right)} .
\end{aligned}
$$

Since in general energy functions are not necessarily known, nor easy to compute in the time domain for complex circuits, we can use quadratic differential forms to facilitate such computations. In the case of the dissipation, this can be easily done in the frequency domain.

4.1. Losses in the Frequency Domain. The integral version of (16) in terms of the auxiliary variable $z$ can be expressed as

$$
\begin{aligned}
& \int_{-\infty}^{+\infty} Q_{\Phi^{\prime}}(z) d t=\int_{-\infty}^{+\infty} Q_{\Delta^{\prime}}(z) d t \\
& \quad=\int_{-\infty}^{+\infty} \frac{1}{2}\left(M\left(\frac{d}{d t}\right) z\right)^{\top}\left[\begin{array}{cc}
0 & I_{m} \\
I_{m} & 0
\end{array}\right] M\left(\frac{d}{d t}\right) z d t
\end{aligned}
$$

then, using Parseval's theorem, we have that

$$
\begin{aligned}
& \int_{-\infty}^{+\infty} Q_{\Phi^{\prime}}(z) d t \\
& \quad=\frac{1}{2 \pi} \int_{-\infty}^{+\infty} \frac{1}{2} \widehat{z}^{\top} M(-j \omega)^{\top}\left[\begin{array}{cc}
0 & I_{m} \\
I_{m} & 0
\end{array}\right] M(j \omega) \widehat{z} d \omega,
\end{aligned}
$$

where $\widehat{z}$ corresponds to the Fourier transform of the auxiliary variable $z$. From this relationship between the time and frequency domain and the fact that $\int_{-\infty}^{+\infty} Q_{\Phi^{\prime}}(z) d t=$ $\int_{-\infty}^{+\infty} Q_{\Delta^{\prime}}(z) d t$ we can conclude that (see proof of Proposition 5.4 of [20])

$$
\Phi^{\prime}(-j \omega, j \omega)=\Delta^{\prime}(-j \omega, j \omega),
$$

which is a convenient way to compute dissipation.

Example 3. Given the circuit in Figure 1, we have that

$$
\begin{aligned}
& M(j \omega) \\
& =\left[\begin{array}{c}
L_{1} L_{2} C(j \omega)^{3}+R L_{1} C(j \omega)^{2}+\left(L_{1}+L_{2}\right) j \omega+R \\
L_{2} C(j \omega)^{2}+R C j \omega+1
\end{array}\right] .
\end{aligned}
$$

Then we compute

$$
\begin{aligned}
\widehat{z}^{\top} \Phi^{\prime}(-j \omega, j \omega) \widehat{z} & =\frac{1}{2} \widehat{z}^{\top} M(-j \omega)^{\top}\left[\begin{array}{ll}
0 & 1 \\
1 & 0
\end{array}\right] M(j \omega) \widehat{z} \\
& =\widehat{i_{2}} R \widehat{i_{2}} .
\end{aligned}
$$

The above equation corresponds to the dissipation

$$
Q_{\Delta^{\prime}}(z)=R i_{2}^{2} .
$$

In a special case when $Q_{\Delta}=0$, the system is called lossless. This case will play a main role in our analysis since, as we later show, it corresponds to the case where conduction losses on SC converters are neglected.

4.2. Energy Storage in Lossless Circuits. The energy stored in a lossless circuit can be easily computed algebraically; we first compute the two-variable polynomial version of $Q_{\Phi^{\prime}}$ as

$$
\Phi^{\prime}(\zeta, \eta):=\frac{1}{2} M(\zeta)^{\top}\left[\begin{array}{cc}
0 & I_{m} \\
I_{m} & 0
\end{array}\right] M(\eta)
$$

Then, according to Section 4.1 if the system is lossless (zero dissipation), that is,

$$
\Phi^{\prime}(-j \omega, j \omega)=\Delta^{\prime}(-j \omega, \omega)=0,
$$

it follows that $Q_{\Phi^{\prime}}=(d / d t) Q_{\Psi^{\prime}}$. We can easily compute $Q_{\Psi^{\prime}}$ in two-variable polynomial terms; that is,

$$
\underbrace{\Phi^{\prime}(\zeta, \eta)}_{Q_{\Phi^{\prime}}}=\underbrace{(\zeta+\eta)}_{d / d t} \underbrace{\Psi^{\prime}(\zeta, \eta)}_{Q_{\Psi^{\prime}}} .
$$

It follows that

$$
\Psi^{\prime}(\zeta, \eta):=\frac{1}{\zeta+\eta} \Phi^{\prime}(\zeta, \eta) .
$$

Then $\Psi^{\prime}(\zeta, \eta)$ induces $Q_{\Psi^{\prime}}$, which corresponds to the stored energy.

The study of lossless systems and the computation of the stored energy is of interest in this paper, since these systems can, almost paradoxically, lose energy if a switching mechanism is involved as in the case of SC converters; we briefly revisit this very well-known issue in the following section using the proposed mathematical framework. 


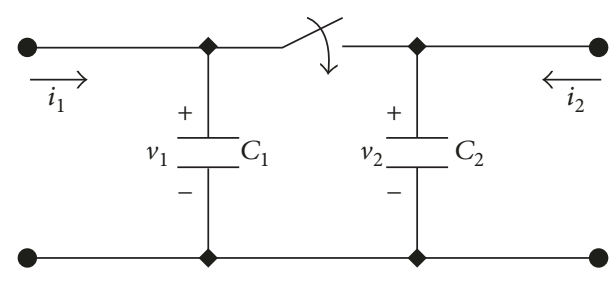

FIgURE 2: Switched-capacitor cell.

\section{Energy Losses in Switched-Capacitors}

We review the problem of determining energy losses that are induced by charging/discharging the capacitors in Figure 2. Using a modeling specification as in (4), before closing the switch, the dynamics of the circuit can be described by

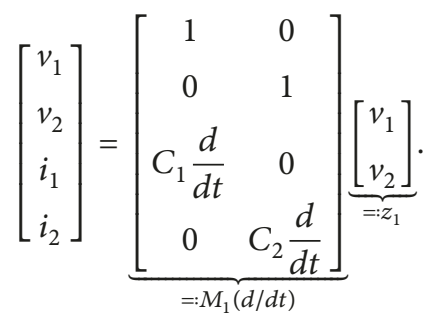

Analogously, after closing the switch, the new dynamics can be described by

$$
\left[\begin{array}{c}
v_{1} \\
i_{1}+i_{2}
\end{array}\right]=\underbrace{\left[\begin{array}{c}
1 \\
\left(C_{1}+C_{2}\right) \frac{d}{d t}
\end{array}\right]}_{=: M_{2}(d / d t)} \underbrace{v_{2}}_{=: z_{2}} .
$$

Consider the following proposition.

Proposition 4. Consider the circuit in Figure 2 where the switch is closed at $t=0$. Define $Q_{\Psi_{1}^{\prime}}$ as the energy stored in the circuit before the switch and $Q_{\Psi_{2}^{\prime}}$ as the one after the switch. Define $\Delta_{\text {loss }}:=Q_{\Psi_{1}^{\prime}}\left(0^{-}\right)-Q_{\Psi_{2}^{\prime}}\left(0^{+}\right)$; therefore,

$$
\Delta_{\text {loss }}=\frac{1}{2} \frac{C_{1} C_{2}}{C_{1}+C_{2}}\left(v_{1}\left(0^{-}\right)-v_{2}\left(0^{-}\right)\right)^{2} .
$$

Proof. In order to prove the claim note first that, using $M_{i}(d / d t), i=1,2$, from (30) and (31), respectively, we can verify that

$$
\begin{gathered}
\frac{1}{2} M_{1}(-j \omega)^{\top}\left[\begin{array}{cc}
0 & I_{2} \\
I_{2} & 0
\end{array}\right] M_{1}(j \omega)=0, \\
\frac{1}{2} M_{2}(-j \omega)^{\top}\left[\begin{array}{ll}
0 & 1 \\
1 & 0
\end{array}\right] M_{2}(j \omega)=0,
\end{gathered}
$$

which implies that each dynamic regime is individually lossless. In order to compute $\Delta_{\text {loss }}$ we use the principle of conservation of charge, which establishes that the total charge within the circuit is the same before and after the switch; that is,

$$
C_{1} v_{1}\left(0^{-}\right)+C_{2} v_{2}\left(0^{-}\right)=C_{1} v_{1}\left(0^{+}\right)+C_{2} v_{2}\left(0^{+}\right) .
$$

Using this equation and taking into account the fact that $v_{1}\left(0^{+}\right)=v_{2}\left(0^{+}\right)$, due to the parallel connection of $C_{1}$ and $C_{2}$, it follows that

$$
v_{1}\left(0^{+}\right)=v_{2}\left(0^{+}\right)=\frac{C_{1} v_{1}\left(0^{-}\right)+C_{2} v_{2}\left(0^{-}\right)}{C_{1}+C_{2}} .
$$

Using quadratic differential forms and (30)-(31), we can compute the stored energy for both subcircuits by using

$$
\begin{aligned}
\Psi_{1}^{\prime}(\zeta, \eta) & :=\frac{1}{\zeta+\eta} M_{1}(\zeta)^{\top}\left[\begin{array}{cc}
0 & I_{2} \\
I_{2} & 0
\end{array}\right] M_{1}(\eta) \\
& =\left[\begin{array}{cc}
\frac{1}{2} C_{1} & 0 \\
0 & \frac{1}{2} C_{2}
\end{array}\right] ; \\
\Psi_{2}^{\prime}(\zeta, \eta) & :=\frac{1}{\zeta+\eta} M_{2}(\zeta)^{\top}\left[\begin{array}{ll}
0 & 1 \\
1 & 0
\end{array}\right] M_{2}(\eta) \\
& =\frac{1}{2}\left(C_{1}+C_{2}\right) .
\end{aligned}
$$

The two-variable polynomials $\Psi_{1}^{\prime}(\zeta, \eta)$ and $\Psi_{2}^{\prime}(\zeta, \eta)$ induce the following QDFs:

$$
\begin{aligned}
& Q_{\Psi_{1}^{\prime}}=\left[\begin{array}{ll}
v_{1} & v_{2}
\end{array}\right]\left[\begin{array}{cc}
\frac{1}{2} C_{1} & 0 \\
0 & \frac{1}{2} C_{2}
\end{array}\right]\left[\begin{array}{l}
v_{1} \\
v_{2}
\end{array}\right], \\
& Q_{\Psi_{2}^{\prime}}=\frac{1}{2}\left(C_{1}+C_{2}\right) v_{2}^{2} .
\end{aligned}
$$

Finally, using (35) to define the value of $v_{2}\left(0^{+}\right)$, we compute $\Delta_{\text {loss }}=Q_{\Psi_{1}^{\prime}}\left(0^{-}\right)-Q_{\Psi_{2}^{\prime}}\left(0^{+}\right)$; that is,

$$
\begin{aligned}
\Delta_{\text {loss }}= & {\left[\begin{array}{ll}
v_{1}\left(0^{-}\right) & v_{2}\left(0^{-}\right)
\end{array}\right]\left[\begin{array}{cc}
\frac{1}{2} C_{1} & 0 \\
0 & \frac{1}{2} C_{2}
\end{array}\right]\left[\begin{array}{l}
v_{1}\left(0^{-}\right) \\
v_{2}\left(0^{-}\right)
\end{array}\right] } \\
& -\frac{1}{2}\left(C_{1}+C_{2}\right)\left(\frac{C_{1} v_{1}\left(0^{-}\right)+C_{2} v_{2}\left(0^{-}\right)}{C_{1}+C_{2}}\right)^{2} .
\end{aligned}
$$

The claim follows by straightforward algebraic reductions.

Equation (32) represents the difference between the energy stored before and after the switch and is usually called charging/discharging loss. In real-life such loss occurs due to the presence of a current between capacitors and its mechanism of dissipation is the parasitic resistance of the loop. It is important to emphasize that, even when such parasitic resistance is neglected, the current appears in the form of a Dirac impulse (cf. $[8,11]$ ), that is induced by the voltage difference between capacitors at the switching instant.

Remark 5. The results presented in this section can be compared to those in $[9,11]$. In our case we obtain the result using the modeling of external dynamics and quadratic 


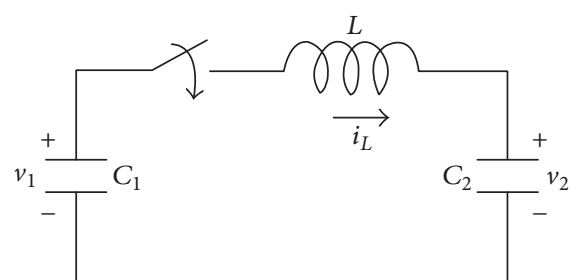

FIGURE 3: Resonant switched-capacitor cell.

differential forms. Our method can be easily extended to more complex converter topologies as exemplified in [7], for which the algorithmic advantages of the polynomial approach can be exploited.

\section{Lossless Resonant Switched-Capacitors}

In this section we show that, unlike traditional SC (inductorless) topologies, the transfer of charge between the capacitors that occurs in the time interval $t=[0, \pi]$ in a resonant cell, as in Figure 3, is lossless. Consider the following proposition.

Proposition 6. Consider the circuit in Figure 3 where Mode 1 corresponds to the circuit configuration when the switch is open and Mode 2 to the one when the switch is closed. Define a zero-current switching signal (cf. [16]) (for ease of exposition we use $\omega t$ as the time axis, where $\omega:=(1 / 2 \pi) \sqrt{\left(C_{1}+C_{2}\right) / C_{1} C_{2} L}$ corresponds to the characteristic frequency of Mode 2) as

$$
s(t)= \begin{cases}\text { Mode } 1, & t<0, \\ \text { Mode 2, } & 0 \leq t \leq \pi \\ \text { Mode } 1, & t>\pi\end{cases}
$$

Define $Q_{\Psi_{1}^{\prime}}$ as the energy storage function of the circuit before the switch and $Q_{\Psi_{2}^{\prime}}$ as the one after the switch. Defining $\Delta_{\text {loss }}:=$ $Q_{\Psi_{1}^{\prime}}(0)-Q_{\Psi_{2}^{\prime}}(\pi)$, it follows that $\Delta_{\text {loss }}=0$.

Proof. We first obtain the linear differential models of the resonant cell in Figure 3 corresponding to Mode 1 before and Mode 2 after the switch, respectively; that is,

$$
\begin{gathered}
\text { Mode 1: }\left\{\begin{array}{l}
C_{1} \frac{d}{d t} v_{1}=0 \\
i_{L}=0 \\
C_{2} \frac{d}{d t} v_{2}=0,
\end{array}\right. \\
\text { Mode 2: }\left\{\begin{array}{l}
C_{1} \frac{d}{d t} v_{1}=-i_{L} \\
L \frac{d}{d t} i_{L}=v_{1}-v_{2} \\
C_{2} \frac{d}{d t} v_{2}=i_{L} .
\end{array}\right.
\end{gathered}
$$

In order to compute $\Delta_{\text {loss }}$, we can use the time domain solution of $\operatorname{col}\left(v_{1}, i_{L}, v_{2}\right)$. Note that $v_{1}$ and $v_{2}$ are continuous and their initial conditions $v_{1}(0)$ and $v_{2}(0)$ are fixed but otherwise arbitrary. Moreover, zero-current switching ensures that $i_{L}$ is equal to zero at every switching instant. Therefore, we can define

$$
x:=\left[\begin{array}{c}
v_{1} \\
i_{L} \\
v_{2}
\end{array}\right]
$$

$$
x\left(0^{-}\right):=\left[\begin{array}{c}
v_{1}\left(0^{-}\right) \\
0 \\
v_{2}\left(0^{-}\right)
\end{array}\right] \text {. }
$$

The time domain solution (cf. [22]) of the dynamic equations of Mode 2 in (40) is given by

$$
x(t)=e^{A t} x\left(0^{-}\right),
$$

where

$$
A=\left[\begin{array}{ccc}
0 & -\frac{1}{C_{1}} & 0 \\
\frac{1}{L} & 0 & -\frac{1}{L} \\
0 & \frac{1}{C_{2}} & 0
\end{array}\right]
$$

Using (42), we obtain

$$
\begin{aligned}
& {\left[\begin{array}{l}
v_{1}(t) \\
i_{L}(t) \\
v_{2}(t)
\end{array}\right]} \\
& =\left[\begin{array}{c}
\frac{1}{2} v_{1}\left(0^{-}\right)+\frac{1}{\frac{2}{2} v_{2}}\left(0^{-}\right)+\frac{1}{2}\left(v_{1}\left(0^{-}\right)-v_{2}\left(0^{-}\right)\right) \cos (t) \\
\sqrt{\frac{C_{\mathrm{eq}}}{L}}\left(v_{1}\left(0^{-}\right)-v_{2}\left(0^{-}\right)\right) \sin (t) \\
\frac{1}{2} v_{1}\left(0^{-}\right)+\frac{1}{2} v_{2}\left(0^{-}\right)+\frac{1}{2}\left(v_{2}\left(0^{-}\right)-v_{1}\left(0^{-}\right)\right) \cos (t)
\end{array}\right],
\end{aligned}
$$

where $C_{\mathrm{eq}}=C_{1} C_{2} /\left(C_{1}+C_{2}\right)$. Now, compute the instantaneous power for both dynamic modes; that is,

$$
\begin{aligned}
Q_{\Phi_{i}^{\prime}}\left(z_{i}\right)=z_{i}^{\top} M_{i}\left(\frac{d}{d t}\right)^{\top}\left[\begin{array}{cc}
0 & I_{2} \\
I_{2} & 0
\end{array}\right] M_{i}\left(\frac{d}{d t}\right) z_{i} & \\
& i=1,2,
\end{aligned}
$$

where $z_{1}:=\operatorname{col}\left(v_{1}, v_{2}\right), z_{2}:=\operatorname{col}\left(v_{1}, i_{L}\right)$, and

$$
M_{1}\left(\frac{d}{d t}\right):=\left[\begin{array}{cc}
1 & 0 \\
0 & 1 \\
C_{1} \frac{d}{d t} & 0 \\
0 & C_{2} \frac{d}{d t}
\end{array}\right]
$$




$$
M_{2}\left(\frac{d}{d t}\right):=\left[\begin{array}{cc}
1 & 0 \\
1 & -L \frac{d}{d t} \\
C_{1} \frac{d}{d t} & 1 \\
C_{2} \frac{d}{d t} & -L C_{2} \frac{d^{2}}{d t^{2}}-1
\end{array}\right]
$$

In polynomial terms it follows that

$$
\Phi_{i}^{\prime}(\zeta, \eta):=\frac{1}{2} M_{i}(\zeta)^{\top}\left[\begin{array}{cc}
0 & I_{2} \\
I_{2} & 0
\end{array}\right] M_{i}(\zeta), \quad i=1,2 .
$$

It can be easily verified that both modes are lossless; that is, $\Phi_{i}^{\prime}(-j \omega, j \omega)=0$ for $i=1,2$. Then we can compute the energy functions:

$$
\begin{aligned}
& \Psi_{1}^{\prime}(\zeta, \eta):=\frac{1}{\zeta+\eta} \Phi_{1}^{\prime}(\zeta, \eta)=\underbrace{\left[\begin{array}{cc}
\frac{1}{2} C_{1} & 0 \\
0 & \frac{1}{2} C_{2}
\end{array}\right]}_{=: \widetilde{\Psi}_{1}} ; \\
& \Psi_{2}^{\prime}(\zeta, \eta):=\frac{1}{\zeta+\eta} \Phi_{2}^{\prime}(\zeta, \eta) \\
& =\left[\begin{array}{ll}
I_{2} & I_{2} \zeta
\end{array}\right] \underbrace{\left[\begin{array}{cccc}
\frac{1}{2}\left(C_{1}+C_{2}\right) & 0 & 0 & -\frac{1}{2} L C_{2} \\
0 & \frac{1}{2} L & 0 & 0 \\
0 & 0 & 0 & 0 \\
-\frac{1}{2} L C_{2} & 0 & 0 & \frac{1}{2} L^{2} C_{2}
\end{array}\right]}_{=: \widetilde{\Psi}_{2}} \text {. }
\end{aligned}
$$

These polynomial matrices induce the following QDFs:

$$
\begin{aligned}
& Q_{\Psi_{1}^{\prime}}:=\left[\begin{array}{ll}
v_{1} & v_{2}
\end{array}\right] \widetilde{\Psi}_{1}\left[\begin{array}{l}
v_{1} \\
v_{2}
\end{array}\right], \\
& Q_{\Psi_{2}^{\prime}}:=\left[\begin{array}{llll}
v_{1} & i_{L} & \frac{d}{d t} v_{1} & \frac{d}{d t} i_{L}
\end{array}\right] \widetilde{\Psi}_{2}\left[\begin{array}{c}
v_{1} \\
i_{L} \\
\frac{d}{d t} v_{1} \\
\frac{d}{d t} i_{L}
\end{array}\right] .
\end{aligned}
$$

In order to compute $\Delta_{\text {loss }}=Q_{\Psi_{1}^{\prime}}(0)-Q_{\Psi_{2}^{\prime}}(\pi)$, we can use (44) to firstly compute

$$
\frac{d}{d t}\left[\begin{array}{l}
v_{1}(t) \\
i_{L}(t) \\
v_{2}(t)
\end{array}\right]=\left[\begin{array}{c}
-\frac{1}{2}\left(v_{1}\left(0^{-}\right)-v_{2}\left(0^{-}\right)\right) \sin (t) \\
\sqrt{\frac{C_{\mathrm{eq}}}{L}}\left(v_{1}\left(0^{-}\right)-v_{2}\left(0^{-}\right)\right) \cos (t) \\
-\frac{1}{2}\left(v_{2}\left(0^{-}\right)-v_{1}\left(0^{-}\right)\right) \sin (t)
\end{array}\right] ;
$$

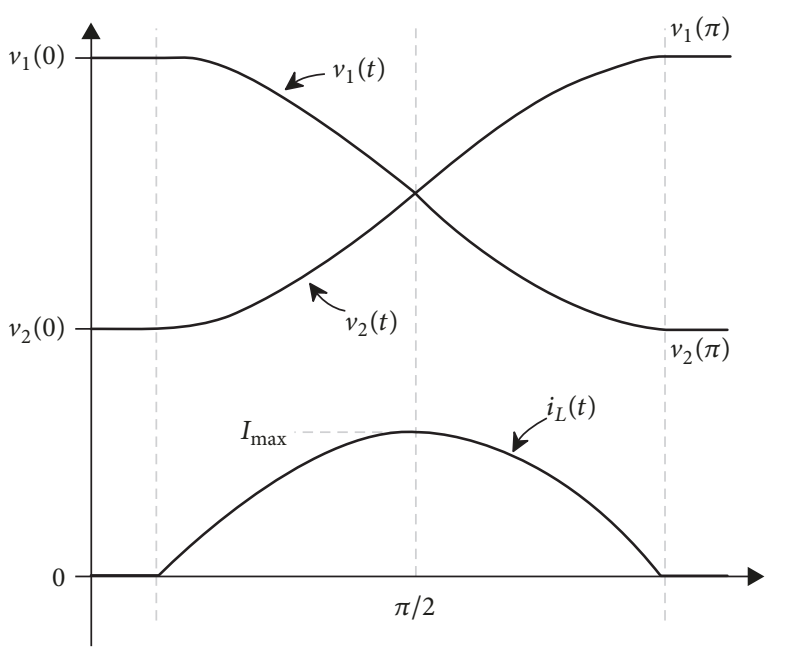

Figure 4: Time domain solution (44) when $v_{1}(0)>v_{2}(0)$.

then it can be easily corroborated using (41), (44), and (50) that

$$
\begin{aligned}
& \Delta_{\text {loss }}=\left[\begin{array}{ll}
v_{1}\left(0^{-}\right) & v_{2}\left(0^{-}\right)
\end{array}\right] \widetilde{\Psi}_{1}\left[\begin{array}{c}
v_{1}\left(0^{-}\right) \\
i v_{2}\left(0^{-}\right)
\end{array}\right]
\end{aligned}
$$

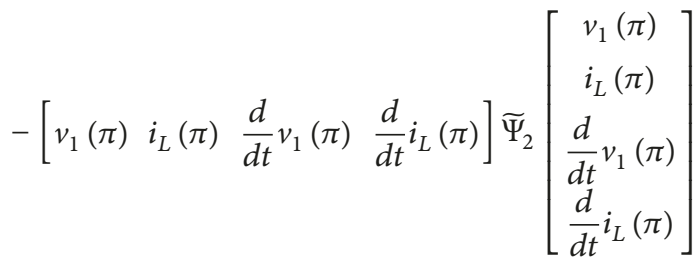

$$
\begin{aligned}
& =0 \text {. }
\end{aligned}
$$

The claim is proved.

By comparing the results of Proposition 6 with respect to those of Proposition 4, we can conclude that the charge transferring mechanism of resonant switched-capacitor converters does not involve charging/discharging losses, since the energy stored before and after the switch is conservative. In the following section we will further explore the relationship between energy storage in resonant SC and pure SC converters.

Remark 7. In Figure 4, we show the traces obtained from a time domain solution (44) for the general case when $v_{1}(0)>$ $v_{2}(0)$, for the interval of time at which the switch is closed. Note that according to Proposition 6 the lossless mechanism in resonant $\mathrm{SC}$ has the zero-current switching as a necessary condition. For instance, if the switch is open when $i_{L} \neq$ 0 , then the energy $(1 / 2) L i_{L}^{2}$ will be lost since it will be instantaneously forced to be zero as well.

\section{Energy Transferring Comparison between Resonant SC and Pure SC Converters}

In this section, we prove that (32) is a physical fundamental quantity in both resonant SC and pure SC converters that 
represents the upper bound of the energy losses. By means of this analysis we open the door to the computation of two relevant performance parameters, the maximum peak current value, and a relative loss factor in resonant converters. Such computation is instrumental to achieve benchmarking specifications during a design process. In order to do so, we first consider the following proposition.

Proposition 8. Under the assumptions of Proposition 6, using the inductor current variable $i_{L}$ and defining $Q_{\text {ind }}:=(1 / 2) L i_{L}^{2}$, it follows that

$$
\max _{0 \leq t \leq \pi}\left\{Q_{\text {ind }}(t)\right\}=\frac{1}{2} \frac{C_{1} C_{2}}{C_{1}+C_{2}}\left(v_{1}\left(0^{-}\right)-v_{2}\left(0^{-}\right)\right)^{2} .
$$

Proof. Consider Mode 2 (when the switch is closed); note that the maximum instantaneous value of $i_{L}$ occurs when $L(d / d t) i_{L}=0$, which occurs according to (44) at $t=\pi / 2$. It follows that the maximum amount of energy stored by the inductor equals

$$
\max _{0 \leq t \leq \pi}\left\{Q_{\text {ind }}(t)\right\}=\frac{1}{2} L\left(i_{L}\left(\frac{\pi}{2}\right)\right)^{2} .
$$

To finish the proof simply substitute $i_{L}$ from (44) evaluated at $\pi / 2$. The claim is proved.

From Proposition 8 we conclude that the maximum level of energy storage in inductors of resonant SC topologies accounts for the energy that would be dissipated by its pure SC counterpart. This result enables the computation of the resonant inductor according to specifications. Let us define the maximum current through the inductor as $I_{\max }:=$ $i_{L}(\pi / 2)$; then according to Proposition 8

$$
\frac{1}{2} L I_{\max }^{2}=\frac{1}{2} \frac{C_{1} C_{2}}{C_{1}+C_{2}} \Delta V^{2}
$$

where $\Delta V:=v_{1}\left(0^{-}\right)-v_{2}\left(0^{-}\right)$. Consequently

$$
L=\frac{C_{1} C_{2}}{C_{1}+C_{2}}\left(\frac{\Delta V}{I_{\max }}\right)^{2},
$$

where the values of $\Delta V$ and the capacitors can be easily determined when the topology is given; see, for example, the parameter selection in [24] and the voltage gain computed in [25]. The value of $I_{\max }$ can be thus defined according to desired specifications for the semiconductor devices (see, e.g., [26], where a peak value is approximated experimentally in switched-capacitor converters). Note that our computation of $L$ can be regarded as a refinement of the results in [26] applied in our case to resonant SC converters.

Finally, we introduce a result that considers the relationship between losses in the presence of parasitic resistances in resonant SC converters and pure SC converters. We show that the losses in such a realistic circuit are bounded by the fundamental quantities $\Delta_{\text {loss }}$ introduced in Propositions 4 and 6. In order to do so, we consider the circuit in Figure 5.

Lemma 9. Consider the circuit in Figure 5 where Mode 1 corresponds to the circuit configuration when the switch is open

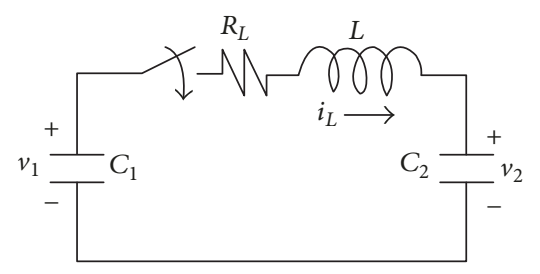

FIGURE 5: Resonant switched-capacitor cell with a parasitic series resistance $R_{L}$.

and Mode 2 to the one when the switch is closed. Assume $i_{L}(0)=0$ and define the switching signal

$$
s(t)= \begin{cases}\text { Mode } 1, & t<0, \\ \text { Mode 2, } & t \in[0, \infty] .\end{cases}
$$

Define $Q_{\Psi_{1}^{\prime}}$ as the energy storage function of the circuit before the switch and $Q_{\Psi_{2}^{\prime}}$ as the one after the switch. Let $\omega$ be the imaginary part of the complex eigenvalues associated with Mode 2. Define

$$
\Delta_{\text {loss }}:=\frac{1}{2} \frac{C_{1} C_{2}}{C_{1}+C_{2}}\left(v_{1}\left(0^{-}\right)-v_{2}\left(0^{-}\right)\right)^{2} .
$$

The following statements hold:

$$
\begin{aligned}
& \text { (1) }\left[L=0, R_{L}=0\right] \Rightarrow\left[Q_{\Psi_{1}^{\prime}}\left(0^{-}\right)-Q_{\Psi_{2}^{\prime}}\left(0^{+}\right)=\Delta_{\text {loss }}\right] . \\
& \text { (2) }\left[L \neq 0, R_{L}=0\right] \Rightarrow\left[Q_{\Psi_{1}^{\prime}}(0)-Q_{\Psi_{2}^{\prime}}(\pi)=0\right] . \\
& \text { (3) }\left[R_{L} / \omega L \rightarrow \infty\right] \Rightarrow\left[Q_{\Psi_{1}^{\prime}}(0)-Q_{\Psi_{2}^{\prime}}(\pi)=\Delta_{\text {loss }}\right] . \\
& \text { (4) }\left[R_{L} / \omega L \rightarrow 0\right] \Rightarrow\left[Q_{\Psi_{1}^{\prime}}(0)-Q_{\Psi_{2}^{\prime}}(\pi)=0\right] .
\end{aligned}
$$

Proof. Statements (1) and (2) follow directly from Propositions 4 and 6 , respectively. In order to prove statements (3) and (4) let us consider the equations of Mode 2; that is,

$$
\text { Mode 2 : }\left\{\begin{array}{l}
C_{1} \frac{d}{d t} v_{1}=-i_{L} \\
L \frac{d}{d t} i_{L}=v_{1}-v_{2}-R_{L} i_{L} \\
C_{2} \frac{d}{d t} v_{2}=i_{L} .
\end{array}\right.
$$

Then considering its time domain solution, that is,

$$
\left[\begin{array}{l}
v_{1} \\
i_{L} \\
v_{2}
\end{array}\right]=e^{A t}\left[\begin{array}{c}
v_{1}(0) \\
0 \\
v_{2}(0)
\end{array}\right]
$$

with

$$
A=\left[\begin{array}{ccc}
0 & -\frac{1}{C_{1}} & 0 \\
\frac{1}{L} & -\frac{R_{L}}{L} & -\frac{1}{L} \\
0 & \frac{1}{C_{2}} & 0
\end{array}\right]
$$




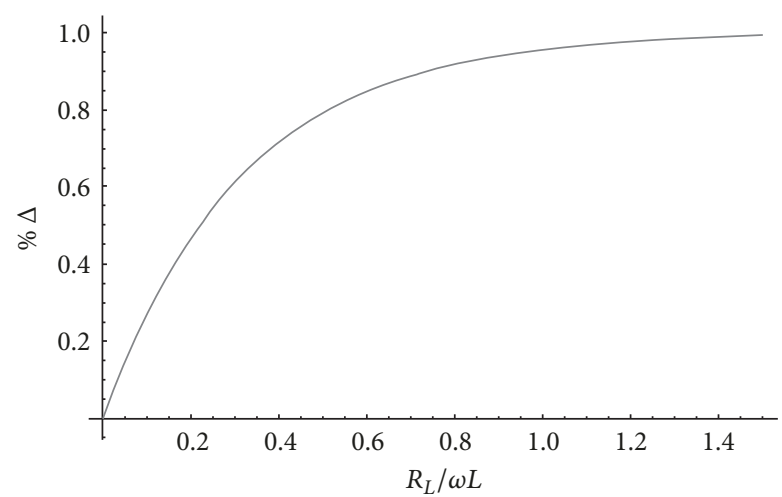

FIgURE 6: Relative loss factor $\% \Delta$ versus the ratio $R_{L} / \omega L$.

and evaluating $Q_{\Psi_{1}^{\prime}}(0)$ and $Q_{\Psi_{2}^{\prime}}(\pi)$ in an analogous way as in the proof of Proposition 6, we obtain

$$
Q_{\Psi_{1}^{\prime}}(0)-Q_{\Psi_{2}^{\prime}}(\pi)=\left(1-e^{-\left(R_{L} / \omega L\right) \pi}\right) \Delta_{\text {loss }} .
$$

Statements (3) and (4) follow readily.

The results of Lemma 9 not only permits generalizing the results obtained along this paper but also provides two important implications: (1) it shows that $\Delta_{\text {loss }}$ is a fundamental physical quantity that bounds the energy losses in both SC and resonant SC converters and (2) it permits developing a relative loss factor that is useful to characterize the efficiency of a resonant converter with parasitic resistances with respect to the worst case scenario. We elaborate the latter point in the following.

Consider the losses in a resonant SC converter with a parasitic series resistance as in the proof of Lemma 9; that is,

$$
\Delta_{\text {res }}:=\left(1-e^{-\left(R_{L} / \omega L\right) \pi}\right) \Delta_{\text {loss }} .
$$

Define the relative loss factor as

$$
\% \Delta:=\frac{\Delta_{\text {res }}}{\Delta_{\text {loss }}}=\left(1-e^{-\left(R_{L} / \omega L\right) \pi}\right),
$$

which represent the percentage of charging/discharging losses with respect to the worst case scenario. Note also that $\% \Delta$ depends on the ratio of the parasitic resistance and the inductive reactance, that is, $R_{L} / \omega L$, which implies that the efficiency of the resonant SC converter also depends on the resonant frequency. We illustrate the relative loss factor with respect to such ratio in Figure 6.

\section{Experimental Results}

Consider the resonant switched-capacitor cell in Figure 3, where $C_{i}:=100 \mu \mathrm{F}, i=1,2, v_{1}(0):=140 \mathrm{~V}, v_{2}(0):=40 \mathrm{~V}$. Moreover, we chose a maximum peak value $I_{\max }=45 \mathrm{~A}$, which according to (55) corresponds to an inductor $L:=$ $246 \mu \mathrm{H}$, which is approximated to a commercial value equal to $250 \mu \mathrm{F}$ in the experiment.

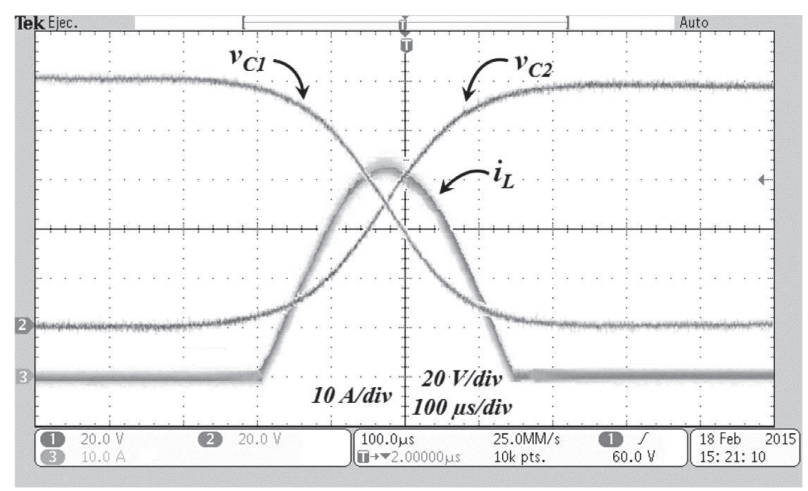

FIGURE 7: Experimental results of the resonant switched-capacitor cell in Figure 3.

In Figure 7 we show the voltage traces as well as the resonant inductor current. It can be noticed that the maximum peak value maintains agreement with the selected value of $I_{\max }=45 \mathrm{~A}$ with a very small error of $1.5 \%$, that is, $44.32 \mathrm{~A}$, which can be associated to the damping induced by conduction losses.

\section{Conclusions}

We showed that resonant SC converters are lossless with respect to charging/discharging processes of capacitors. This analytical proof is supported by the use of a linear and quadratic differential framework, which is introduced for SC converters in this paper. We showed that the maximum energy storage in the resonant inductor equals the dissipated energy by its pure SC counterpart. We also generalize the results by involving a realistic scenario where parasitic resistances are involved. We also develop a relative loss factor that permits designing resonant SC cells according to efficiency specifications. Moreover, we showed theoretically and experimentally that the resonant inductors can be computed with respect to their maximum stored energy and/or their maximum current value. The results presented in this paper indicate that migration from pure SC converter topologies to their resonant counterpart is the most compelling solution to well-known disadvantages such as high peak currents among capacitors and charging losses.

\section{Conflicts of Interest}

The authors declare that they have no conflicts of interest.

\section{References}

[1] C.-K. Cheung, S.-C. Tan, C. K. Tse, and A. Ioinovici, "On energy efficiency of switched-capacitor converters," IEEE Transactions on Power Electronics, vol. 28, no. 2, pp. 862-876, 2013.

[2] D. F. Cortez, G. Waltrich, J. Fraigneaud, H. Miranda, and I. Barbi, "DC-DC converter for dual-voltage automotive systems based on bidirectional hybrid switched-capacitor architectures," IEEE Transactions on Industrial Electronics, vol. 62, no. 5, pp. 3296-3304, 2015. 
[3] S. Kiratipongvoot, S.-C. Tan, and A. Ioinovici, "Phase-shift interleaving control of variable-phase switched-capacitor converters," IEEE Transactions on Industrial Electronics, vol. 60, no. 12, pp. 5575-5584, 2013.

[4] M. D. Vecchia, T. B. Lazzarin, and I. Barbi, "A three-phase AC-AC converter in open-delta connection based on switched capacitor principle," IEEE Transactions on Industrial Electronics, vol. 62, no. 10, pp. 6035-6041, 2015.

[5] G. Wu, X. Ruan, and Z. Ye, "Nonisolated high step-up DC-DC converters adopting switched-capacitor cell," IEEE Transactions on Industrial Electronics, vol. 62, no. 1, pp. 383-393, 2015.

[6] P. J. Mosterman and G. Biswas, "A theory of discontinuities in physical system models," Journal of The Franklin Institute, vol. 335, no. 3, pp. 401-439, 1998.

[7] J. C. Mayo-Maldonado, J. C. Rosas-Caro, and P. Rapisarda, "Modeling approaches for DC-DC converters with switched capacitors," IEEE Transactions on Industrial Electronics, vol. 62, no. 2, pp. 953-959, 2015.

[8] M. Evzelman and S. Ben-Yaakov, "Average-current-based conduction losses model of switched capacitor converters," IEEE Transactions on Power Electronics, vol. 28, no. 7, pp. 3341-3352, 2013.

[9] R. Frasca, M. K. Camlibel, I. C. Goknar, L. Iannelli, and F. Vasca, "Linear passive networks with ideal switches: consistent initial conditions and state discontinuities," IEEE Transactions on Circuits and Systems I: Regular Papers, vol. 57, no. 12, pp. 3138-3151, 2010.

[10] M. D. Seeman and S. R. Sanders, "Analysis and optimization of switched-capacitor DC-DC converters," IEEE Transactions on Power Electronics, vol. 23, no. 2, pp. 841-851, 2008.

[11] C. K. Tse, S. C. Wong, and M. H. L. Chow, "On lossless switched-capacitor power converters," IEEE Transactions on Power Electronics, vol. 10, no. 3, pp. 286-291, 1995.

[12] R. Beiranvand, "Analysis of a switched-capacitor converter above its resonant frequency to overcome voltage regulation issue of resonant SCCs," IEEE Transactions on Industrial Electronics, vol. 63, no. 9, pp. 5315-5325, 2016.

[13] A. Cervera, M. Evzelman, M. M. Peretz, and S. S. Ben-Yaakov, "A high-efficiency resonant switched capacitor converter with continuous conversion ratio," IEEE Transactions on Power Electronics, vol. 30, no. 3, pp. 1373-1382, 2015.

[14] A. Cervera and M. M. Peretz, "Resonant switched-capacitor voltage regulator with ideal transient response," IEEE Transactions on Power Electronics, vol. 30, no. 9, pp. 4943-4951, 2015.

[15] Y. Lei and R. C. N. Pilawa-Podgurski, "A general method for analyzing resonant and soft-charging operation of switchedcapacitor converters," IEEE Transactions on Power Electronics, vol. 30, no. 10, pp. 5650-5664, 2015.

[16] J. C. Rosas-Caro, J. C. Mayo-Maldonado, A. ValderrabanoGonzalez, F. Beltran-Carbajal, J. M. Ramirez-Arredondo, and J. R. Rodriguez-Rodriguez, "DC-DC multiplier boost converter with resonant switching," Electric Power Systems Research, vol. 119, pp. 83-90, 2015.

[17] J. C. Mayo-Maldonado and P. Rapisarda, "Dissipative switched linear differential systems," Institute of Electrical and Electronics Engineers Transactions on Automatic Control, vol. 61, no. 12, pp. 3813-3825, 2016.

[18] J. C. Mayo-Maldonado and P. Rapisarda, "On positive-realness and Lyapunov functions for switched linear differential systems," Institute of Electrical and Electronics Engineers Transactions on Automatic Control, vol. 61, no. 8, pp. 2239-2244, 2016.
[19] J. C. Mayo-Maldonado, P. Rapisarda, and P. Rocha, "Stability of switched linear differential systems," Institute of Electrical and Electronics Engineers Transactions on Automatic Control, vol. 59, no. 8, pp. 2038-2051, 2014.

[20] J. C. Willems and H. L. Trentelman, "On quadratic differential forms," SIAM Journal on Control and Optimization, vol. 36, no. 5, pp. 1703-1749, 1998.

[21] S. R. Pasternak, M. H. Kiani, J. S. Rentmeister, and J. T. Stauth, "Modelling and performance limits of switched-capacitor DCDC converters capable of resonant operation with a single inductor," IEEE Journal of Emerging and Selected Topics in Power Electronics, vol. 5, no. 4, pp. 1746-1760, 2017.

[22] J. Polderman and J. Willems, Introduction to Mathematical System Theory: A Behavioral Approach, Springer, Berlin, Germany, 1997.

[23] J. C. Willems and H. L. Trentelman, "Synthesis of dissipative systems using quadratic differential forms," Institute of Electrical and Electronics Engineers Transactions on Automatic Control, vol. 47, no. 1, pp. 53-69, 2002.

[24] J. Valdez-Reséndiz, A. Claudio-Sánchez, J. Rosas-Caro, G. Guerrero-Ramírez, J. Mayo-Maldonado, and A. Tapia-Hernán$\mathrm{dez}$, "Resonant switched capacitor voltage multiplier with interleaving capability," Electric Power Systems Research, vol. 133, pp. 365-372, 2016.

[25] J. C. Rosas-Caro, J. C. Mayo-Maldonado, F. Mancilla-David, A. Valderrabano-Gonzalez, and F. B. Carbajal, "Single-inductor resonant switched capacitor voltage multiplier with safe commutation," IET Power Electronics, vol. 8, no. 4, pp. 507-516, 2015.

[26] F. Zhang, L. Du, F. Z. Peng, and Z. Qian, "A new design method for high-power high-efficiency switched-capacitor DCDC converters," IEEE Transactions on Power Electronics, vol. 23, no. 2, pp. 832-840, 2008. 


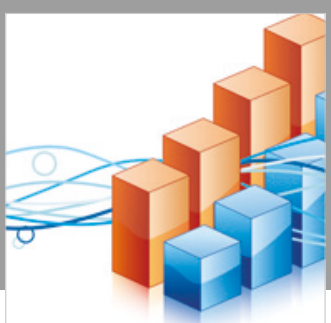

Advances in

Operations Research

\section{-n-m}
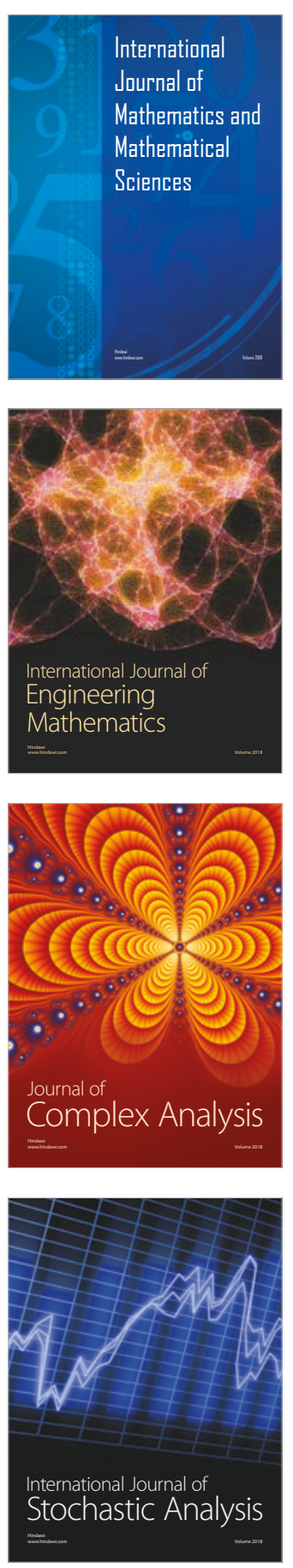
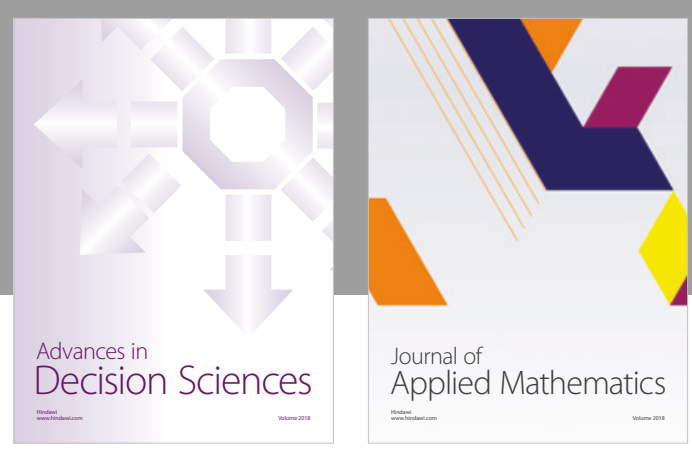

Journal of

Applied Mathematics
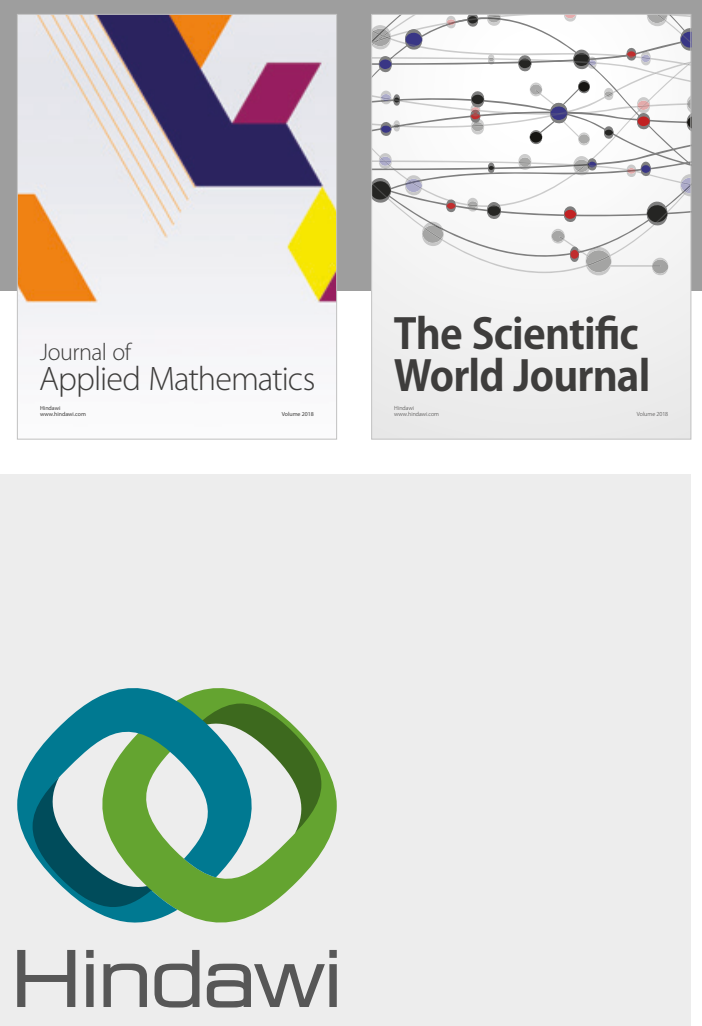

Submit your manuscripts at

www.hindawi.com

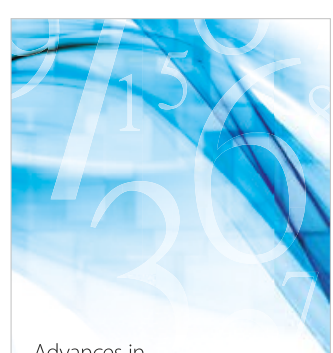

Advances in
Numerical Analysis
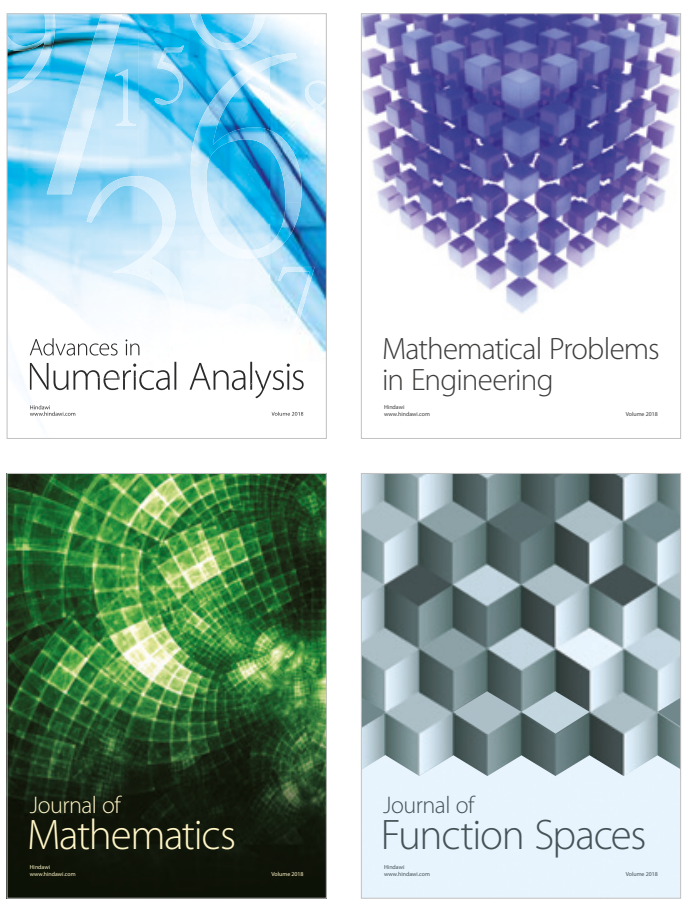

Mathematical Problems in Engineering

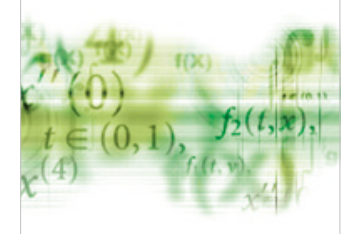

International Journal of

Differential Equations

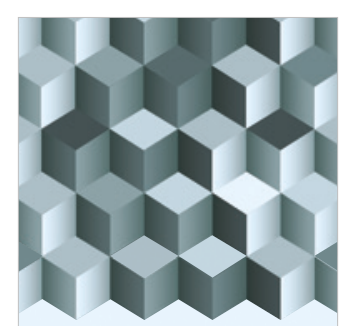

Journal of

Function Spaces

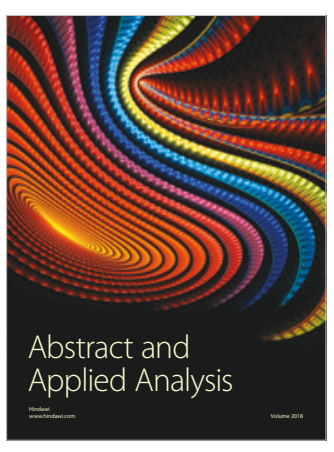

The Scientific

World Journal

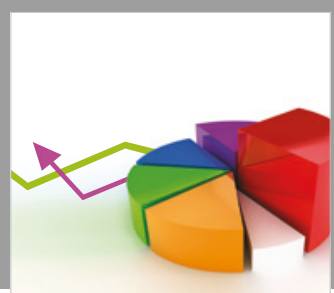

Journal of

Probability and Statistics
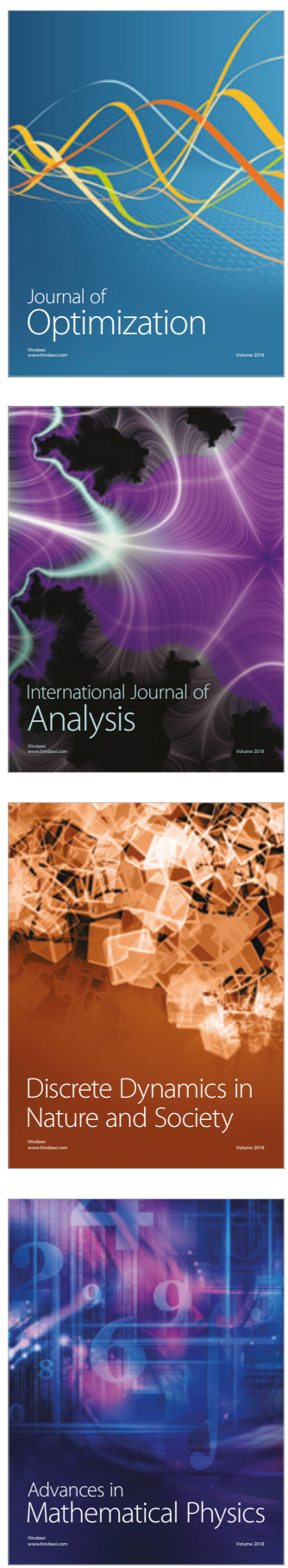